Available online at www.eccomasproceedia.org

Eccomas Proceedia COMPDYN (2021) 1793-1802

ECCOMAS

Proceedia
COMPDYN 2021

$8^{\text {th }}$ ECCOMAS Thematic Conference on Computational Methods in Structural Dynamics and Earthquake Engineering M. Papadrakakis, M. Fragiadakis (eds.) Streamed from Athens, Greece, 28 - 30 June 2021

\title{
DIAGRID SYSTEM COUPLED WITH SHEAR WALLS: ANALYTICAL INVESTIGATION ON THE DYNAMICAL RESPONSE IN TALL BUILDINGS
}

\author{
G. Lacidogna $^{1 *}$, G. Nitti $^{1}$, D. Scaramozzino ${ }^{1}$, and A. Carpinteri ${ }^{1}$ \\ ${ }^{1}$ Department of Structural, Geotechnical and Building Engineering, Politecnico di Torino, Corso Duca \\ degli Abruzzi 24, 10129, Torino, Italy \\ e-mail: giuseppe.lacidogna@polito.it, giuseppe.nitti@polito.it, domenico.scaramozzino@polito.it, \\ alberto.carpinteri@polito.it
}

*Corresponding author: giuseppe.lacidogna@polito.it

\begin{abstract}
Diagrids are tubular structural systems made up of mega-diagonals arranged in a triangular or tetrahedral pattern, which are placed all over the external surface of the building and usually span across several floors. In the last two decades, diagrids have experienced a remarkable development as efficient structural systems in tall building design and construction. This was mainly due to their high lateral stiffness, capability to realize complex-shaped structures and obtain impressive aesthetic results, flexibility of the external diagonals' layout, etc. The structural analysis of these systems is usually carried out by the Finite Element Method (FEM) or by numerical calculations based on simplified assumptions. Recently, we developed a matrix-based method (MBM) to perform the structural analysis of diagrid systems under static forces. The MBM was then coupled with an analytical formulation developed in the past years by some of the authors, the so-called General Algorithm (GA), in order to study the structural response of an external diagrid tube coupled with an internal shear wall under lateral and torque static actions. The study allowed to investigate the influence of the diagonal inclination on the lateral and torsional flexibility of the diagrid-core system. In the past years, the analytical formulation of the GA was also made suitable to analyze the response of threedimensional tall building in the dynamic regime. In this contribution, we show new results regarding the dynamical behavior of an external diagrid structural system coupled with an internal shear wall, as obtained from the GA calculations. Modal analysis was carried out in order to obtain the natural frequencies and mode shapes of the tall building, and the influence of the diagonal inclination on the results was also investigated. Moreover, by applying different harmonic oscillations at different frequencies at the base of the building, the damped dynamical response of the different diagrid-core systems was investigated.
\end{abstract}

Keywords: diagrid, matrix-based method, general algorithm, dynamic analysis, analytical method. 


\section{INTRODUCTION}

In the last decades, the field of tall buildings has undergone a major development due to the introduction of new structural systems able to resist lateral loads very efficiently [1]. Among these, diagrid systems have been exploited worldwide due to their high lateral stiffness and aesthetic potential. The diagrid is a tubular structure placed over the external surface of the building, made up of inclined mega-diagonals [2,3] or tetrahedral configuration [4] which are designed to withstand both lateral and vertical loads. Therefore, in diagrid structures the conventional vertical columns are completely removed from the external tube, as the gravitational loads can be directly transferred to the ground via the mega-diagonals.

Besides the conventional application of Finite Element (FE) calculations, a variety of analytical and simplified methodologies have been proposed in the last years for the structural analysis of diagrid systems under lateral loads. All these methods usually rely on the following basic assumptions: (1) the deformation mechanism of the building is governed by the axial elongation and shortening of the mega-diagonals, (2) the stress-strain relationship of the diagonals lies within the linear elastic regime, and (3) the floor slabs of the building remain plane after deformation. Based on these assumptions, Moon et al. [5] developed a stiffnessbased methodology for the structural analysis and preliminary design of rectangular diagrid tubes under horizontal forces. Mele et al. [6] proposed a hand-based method for the calculation of axial stresses in the diagonals under both lateral and vertical loads. Liu and Ma [7] suggested a modular method for the structural analysis of arbitrary polygonal diagrid tubes, where the shear and bending stiffness of the diagrid modules were analytically calculated.

More recently, we developed a stiffness-based approach based on matrix calculus, which was called the matrix-based method (MBM), for the analysis of generic diagrid systems [8]. The MBM is based on the direct calculation of the global stiffness matrix of the diagrid, by considering the kinematic and equilibrium equations at the level of the rigid floors. The MBM allows to perform very quickly the structural analysis of diagrids under both lateral, vertical and torsional actions. Furthermore, the MBM has been recently integrated within a more general semi-analytical framework, the so-called General Algorithm (GA). The GA has been developed by some of the authors in the last decades and allows to investigate the structural behavior of three-dimensional buildings made up of several vertical resisting elements, such as frames, tubes and shear walls [9-12]. Very recently, we have inserted the MBM within the GA environment in order to perform the structural analysis of an external diagrid tube coupled with internal shear walls [13].

One of the main interesting features of diagrid structures is that their structural response is significantly affected by the layout of external diagonals. This means that the diagonal layout can undergo optimization processes, finding the optimal diagonal arrangement to optimize certain responses of the building. Moon [14] has early shown that there exists an optimal inclination of the external diagonals able to minimize the lateral building deflection under horizontal loads. Various diagonal layouts have been proposed in the following years and the corresponding building efficiency under lateral loads has been investigated [15-18]. Attention has also been paid to torsional actions and the role that these might have in the parametric investigation of the diagrid structural response [13].

In this contribution, we make use of the MBM coupled with the GA in order to investigate how the dynamic response of an external diagrid tube coupled with an internal closed-section shear wall changes according to a change in the inclination of the external diagrid diagonals. The natural frequencies and fundamental mode shapes of the building are extracted by solving the multi-degree-of-freedom (MDOF) unforced undamped equations of motion. The analysis of the natural frequencies and mode shapes of the buildings with different inclinations of the 
diagonals reveals the influence of the diagrid geometry on the overall dynamic response of the system. Furthermore, the damped dynamic response of the diagrid-core structure is also studied under the application of harmonic oscillations of the base of the building. Comparisons between the obtained maximum displacements, velocities, accelerations, inter-story drifts and base shear values are finally made.

\section{METHODOLOGY}

As shown in [13], the MBM can be coupled to the GA in order to carry out the structural analysis of a diagrid-core system under lateral and torsional static actions. More details about the basic assumptions and the applications of the MBM and the GA can be found in [8-13]. In the GA environment the building is assumed to be subjected to two horizontal forces (along the horizontal $\mathrm{X}$ and $\mathrm{Y}$ axes) and one torque moment (around the vertical $\mathrm{Z}$ axis) acting at the level of the floor centroids. It follows that the total number of DOFs in the GA is equal to $3 \mathrm{~N}$, $\mathrm{N}$ being the total number of floors.

In this paper, the MBM-GA framework has been adapted in order to investigate the dynamic response of a diagrid-core system. First of all, the natural frequencies and fundamental mode shapes of the building are evaluated according to the MDOF undamped unforced equations of motion. Then, we apply harmonic oscillations at various frequencies at the base of the building and solve the dynamical problem based on the MDOF forced oscillations in presence of damping.

The first problem can be formulated in simple mathematical terms via the following matrix relationship related to the modal eigenvalue-eigenvector solution:

$$
\left(\mathbf{K}-\omega_{\mathrm{n}}^{2} \mathbf{M}\right) \mathbf{u}_{\mathbf{n}}=\mathbf{0}
$$

where $\mathbf{K}$ and $\mathbf{M}$ represent the stiffness and mass matrix of the structure, $\mathbf{u}_{\mathbf{n}}$ is the massweighted eigenvector associated to the $n$-th mode shape $(n=1,2, \ldots, 3 N)$ and $\omega_{n}$ is the angular frequency associated to the $n$-th mode of vibration. The angular frequency $\omega_{n}$ is related to the vibrational frequency $f_{n}$ and period of vibration $T_{n}$ by $\omega_{n}=2 \pi f_{n}=2 \pi / T_{n}$. More details about the calculation of the stiffness matrix $\mathbf{K}$ and mass matrix $\mathbf{M}$ can be found in $[9,11]$. After the construction of these matrices, equation (1) immediately yields the natural frequencies and mode shapes of the structure.

After evaluating the fundamental vibrational features of the diagrid-core building (frequencies and mode shapes), we also want to investigate the dynamic response of the structure under the application of harmonic oscillations at the base of the structure. This can be done by considering the complete dynamic equation, which includes the contribution of inertia, damping, elastic and external forces:

$$
\mathbf{M} \boldsymbol{\delta}^{\prime \prime}(\mathrm{t})+\mathbf{C} \boldsymbol{\delta}^{\prime}(\mathrm{t})+\mathbf{K} \boldsymbol{\delta}(\mathrm{t})=\mathbf{F}_{\text {ext }}(\mathrm{t})
$$

where $\mathbf{C}$ is the damping matrix of the structure, $\mathbf{F}_{\text {ext }}(\mathrm{t})$ is the vector of external dynamic forces and $\boldsymbol{\delta}^{\prime \prime}(\mathrm{t}), \boldsymbol{\delta}^{\prime}(\mathrm{t})$ and $\boldsymbol{\delta}(\mathrm{t})$ represent the vectors of floor accelerations, velocities and displacements (the apex ' stands for the time-derivative). One can then separate the spatial and temporal contribution within the dynamic displacements $\boldsymbol{\delta}(\mathrm{t})$, as $\boldsymbol{\delta}(\mathrm{t})=\mathbf{U} \mathbf{p}(\mathrm{t})$, where $\mathbf{U}$ is the matrix whose columns contain the time-independent mode shapes un obtained from equation (1) and $\mathbf{p}(\mathrm{t})$ is the vector containing the time-dependent principal coordinates associated to each mode shape. Hence, we obtain:

$$
M \mathbf{U p}^{\prime \prime}(\mathrm{t})+\mathbf{C U} \mathbf{p}^{\prime}(\mathrm{t})+\mathbf{K U p}(\mathrm{t})=\mathbf{F}_{\text {ext }}(\mathrm{t})
$$


Multiplying each term of equation (3) by $\mathbf{U}^{\mathbf{T}}$ and remembering that, due to the massnormalization of $\mathbf{U}, \mathbf{U}^{\mathbf{T}} \mathbf{M} \mathbf{U}=\mathbf{I}$ and $\mathbf{U}^{\mathbf{T}} \mathbf{K} \mathbf{U}=\boldsymbol{\Omega}, \mathbf{I}$ being the identity matrix and $\boldsymbol{\Omega}$ the matrix containing the eigenvalues $\omega_{\mathrm{n}}^{2}$ on the diagonal and zero outside, it follows that:

$$
\mathbf{p}^{\prime \prime}(\mathrm{t})+\mathbf{U}^{\mathrm{T}} \mathbf{C} \mathbf{U p}^{\prime}(\mathrm{t})+\boldsymbol{\Omega} \mathbf{p}(\mathrm{t})=\mathbf{U}^{\mathrm{T}} \mathbf{F}_{\text {ext }}(\mathrm{t})
$$

The problem can now be decoupled with respect to each individual mode $\mathrm{n}$ if one assumes that the matrix $\mathbf{U}^{\mathrm{T}} \mathbf{C} \mathbf{U}$ is diagonal, with diagonal elements equal to $2 \xi_{\mathrm{n}} \omega_{\mathrm{n}}$, where $\xi_{\mathrm{n}}$ represents the damping ratio associated to the $n$-th mode of vibration. Then, one finally obtains the set of $3 \mathrm{~N}$ scalar equations whose unknowns are the temporal principal coordinates $\mathrm{p}_{\mathrm{n}}(\mathrm{t})$ :

$$
\mathrm{p}_{\mathrm{n}}^{\prime \prime}(\mathrm{t})+2 \xi_{\mathrm{n}} \omega_{\mathrm{n}} \mathrm{p}_{\mathrm{n}}{ }^{\prime}(\mathrm{t})+\omega_{\mathrm{n}}{ }^{2} \mathrm{p}_{\mathrm{n}}(\mathrm{t})=\mathbf{u}_{\mathrm{n}}{ }^{\mathrm{T}} \mathbf{F}_{\text {ext }}(\mathrm{t})
$$

Since we apply harmonic oscillations at the base of the building, the vector of external forces $\mathbf{F}_{\text {ext }}(\mathrm{t})$ can be simply written as $\mathbf{F}_{\text {ext }}(\mathrm{t})=\mathbf{M u} \mathbf{u}^{\prime \prime}(\mathrm{t})=\mathbf{M} \mathbf{u g}_{\mathrm{g}}{ }^{\prime} \sin \left(\omega_{\mathrm{g}} \mathrm{t}\right)$, where $\mathbf{u g}_{\mathrm{g}}{ }^{\prime \prime}$ is the vector associated to the ground acceleration and $\omega_{\mathrm{g}}$ the angular frequency of the ground oscillation. The solution of the single-degree-of-freedom (SDOF) equation of motion (5), with the application of the initial resting conditions, leads to:

$$
p_{n}(t)=e^{-\xi_{n} \omega_{n} t}\left[A_{n} \cos \left(\omega_{d, n} t\right)+B_{n} \sin \left(\omega_{d, n} t\right)\right]+C_{n} \cos \left(\omega_{g} t+\phi_{n}\right)
$$

where $\omega_{\mathrm{d}, \mathrm{n}}=\omega_{\mathrm{d}}\left(1-\xi_{\mathrm{n}}^{2}\right)$ is the reduced frequency of the $\mathrm{n}$-th mode of vibration due to its damping, and the coefficients $A_{n}, B_{n}, C_{n}$ and $\phi_{n}$ take the following form:

$$
\left\{\begin{array}{l}
\mathrm{A}_{\mathrm{n}}=-\mathrm{C}_{\mathrm{n}} \sin \left(\phi_{\mathrm{n}}\right) \\
\mathrm{B}_{\mathrm{n}}=\frac{\xi_{\mathrm{n}} \omega_{\mathrm{n}} \mathrm{A}_{\mathrm{n}}-\mathrm{C}_{\mathrm{n}} \omega_{\mathrm{n}} \cos \left(\phi_{\mathrm{n}}\right)}{\omega_{\mathrm{d}, \mathrm{n}}} \\
\mathrm{C}_{\mathrm{n}}=\frac{\mathbf{u}_{\mathrm{n}}^{\mathrm{T}} \mathbf{M u}_{\mathrm{g}}{ }^{\prime \prime}}{\omega_{\mathrm{n}}{ }^{2} \sqrt{\left(1-\beta_{\mathrm{n}}{ }^{2}\right)^{2}+\left(2 \xi_{\mathrm{n}} \beta_{\mathrm{n}}\right)^{2}}} \\
\phi_{\mathrm{n}}=-\arctan \left(\frac{2 \xi_{\mathrm{n}} \beta_{\mathrm{n}}}{1-\beta_{\mathrm{n}}^{2}}\right)
\end{array}\right.
$$

where $\beta_{\mathrm{n}}=\omega_{\mathrm{g}} / \omega_{\mathrm{n}}$ is the ratio between the angular frequency of the external excitation and the one associated to the $\mathrm{n}$-th mode shape. Once all the $3 \mathrm{~N}$ principal coordinates are evaluated from equations (6-7), the displacements of the structure $\boldsymbol{\delta}(\mathrm{t})$ are immediately obtained as $\boldsymbol{\delta}(\mathrm{t})$ $=\mathbf{U p}(\mathrm{t})$. Known the time-dependent displacements of the building floors one can easily find the velocities $\boldsymbol{\delta}^{\prime}(\mathrm{t})$ and accelerations $\boldsymbol{\delta}^{\prime \prime}(\mathrm{t})$ by single and double time-differentiation. The interstory drifts can also be evaluated directly from the vector $\boldsymbol{\delta}(\mathrm{t})$. Finally, the total timedependent base shear $\mathrm{V}(\mathrm{t})$ is calculated by summing all the horizontal elastic forces at the level of the floors, which can be computed as $\mathbf{F}_{\mathbf{e l}}(\mathrm{t})=\mathbf{K} \boldsymbol{\delta}(\mathrm{t})$.

The analytical procedure described in the above paragraphs is applied to a tall building made up of an external steel diagrid tube coupled with an internal closed-section concrete core. Figure 1a shows the dimensions (in meters) of the building plane, while figure $1 \mathrm{~b}$ reports the six different lateral views by considering six different inclinations of the external diagonals [13]. The building has 40 stories and the inter-story height is equal to 4 meters, leading to a total height of the building of 160 meters. The internal core is a $9 \mathrm{~m} \times 9 \mathrm{~m}$ closedsection shear wall made of concrete (Young's modulus of $30 \mathrm{GPa}$ ) and $0.80 \mathrm{~m}$ thick. The ex- 
ternal diagonals are made of steel (Young's modulus of $210 \mathrm{GPa}$ ) and their cross-sectional area is equal to $0.1 \mathrm{~m}^{2}$ [13]. The different diagonal inclinations of the models (figure $1 \mathrm{~b}$ ) range from $32.6^{\circ}$ (model 1) up to $81.1^{\circ}$ (model 6). The mass of the structure is calculated assuming a dead load for the floors equal to $7 \mathrm{kN} / \mathrm{m}^{2}$. Finally, the damping ratio $\xi_{\mathrm{n}}$ is considered equal to 0.05 for all the modes of vibration.

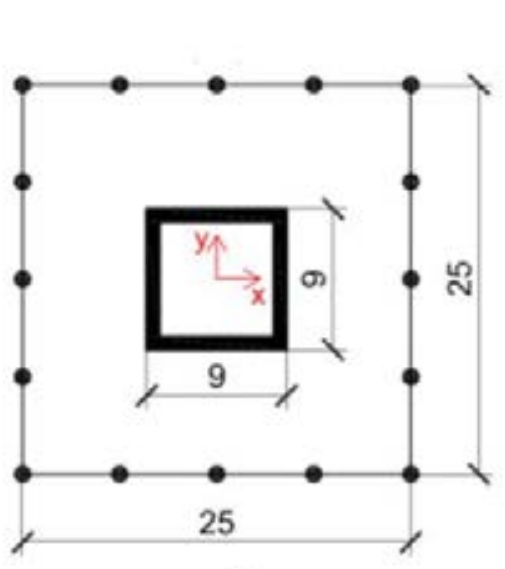

(a)
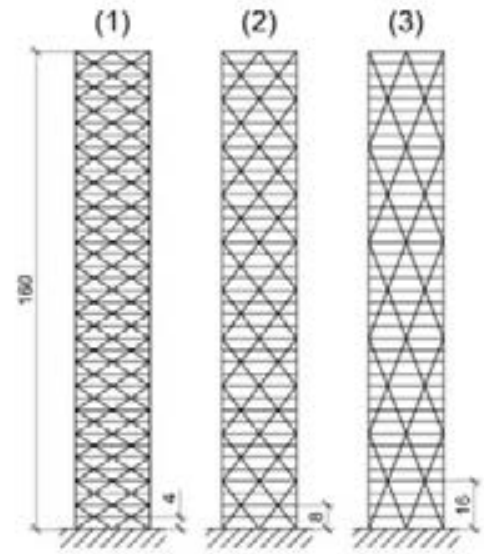

(5)
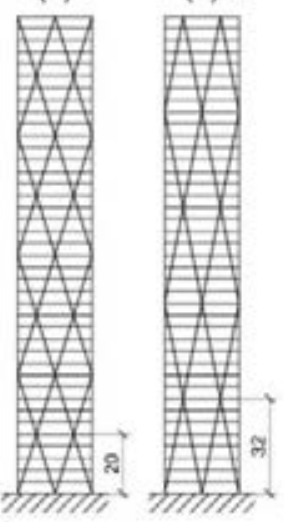

(b)

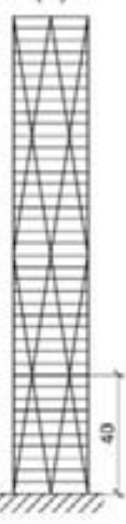

Figure 1: Building geometry: (a) floor plan; (b) lateral views. From Lacidogna et al. [13].

\section{RESULTS AND DISCUSSION}

Table 1 shows the first ten values of the natural frequencies (in $\mathrm{Hz}$ ) arising from the MDOF unforced oscillation problem reported in equation (1) for the six models. The letters X, $\mathrm{Y}$ and $\mathrm{Z}$ in the parentheses describe the predominance direction of the mode shape: $\mathrm{X}$ and $\mathrm{Y}$ refer to lateral oscillations of the building along the $\mathrm{X}$ and $\mathrm{Y}$ direction, respectively, while $\mathrm{T}$ means that there is a not negligible contribution due to the torsional rotation of the building.

As can be seen, due to the symmetry of the building (figure 1a), the first two modes involve lateral oscillations along the $\mathrm{X}$ and $\mathrm{Y}$ axis at the same frequency for all models. The absolute value of this fundamental frequency is found to be maximum $(0.364 \mathrm{~Hz})$ for model 3 and minimum $(0.243 \mathrm{~Hz})$ for model 1 . The first frequency of model $4(0.362 \mathrm{~Hz})$ is close to that of model 3, whereas the first frequencies of models $2(0.321 \mathrm{~Hz}), 5(0.339 \mathrm{~Hz})$ and 6 $(0.320 \mathrm{~Hz})$ lie in between. Since higher frequencies are associated to stiffer responses, these results reflect a feature already found from the static analysis performed in [13], i.e. model 1 (the one with the shallowest diagonals) has the lowest lateral rigidity, whereas models 3 and 4 (with diagonal inclinations around $70^{\circ}$ ) are the stiffest under lateral loads.

For higher mode numbers an important torsional contribution is found to appear associated with displacements of the structure along the Y direction. It is interesting to notice how the first mode shapes involving significant torsional rotations are found at the fifth mode for models 1 and 2 and at the third mode for the other models. Moreover, the fundamental frequency values associated to these first torsional oscillations are found to decrease as the number of the model increases, i.e. it is maximum for model $1(2.313 \mathrm{~Hz})$ and it decreases up to the minimum value for model $6(1.186 \mathrm{~Hz})$. Again, since higher frequencies correspond to stiffer responses, these results show that increasing the diagonal inclination of the diagrid leads to higher torsional flexibility, as already shown by the static analysis reported in [13]. 


\begin{tabular}{ccccccc}
\hline \multirow{2}{*}{ Mode number } & \multirow{2}{*}{ Model 1 } & Model 2 & Model 3 & Model 4 & \multirow{2}{*}{ Model 5 } & \multirow{2}{*}{ Model6 } \\
\hline 1 & $0.243(\mathrm{X})$ & $0.321(\mathrm{X})$ & $0.364(\mathrm{X})$ & $0.362(\mathrm{X})$ & $0.339(\mathrm{X})$ & $0.320(\mathrm{Y})$ \\
2 & $0.243(\mathrm{Y})$ & $0.321(\mathrm{Y})$ & $0.364(\mathrm{Y})$ & $0.362(\mathrm{Y})$ & $0.399(\mathrm{Y})$ & $0.320(\mathrm{X})$ \\
3 & $1.499(\mathrm{X})$ & $1.857(\mathrm{Y})$ & $1.581(\mathrm{Y}+\mathrm{T})$ & $1.442(\mathrm{Y}+\mathrm{T})$ & $1.243(\mathrm{Y}+\mathrm{T})$ & $1.186(\mathrm{Y}+\mathrm{T})$ \\
4 & $1.499(\mathrm{Y})$ & $1.857(\mathrm{X})$ & $1.834(\mathrm{X})$ & $1.746(\mathrm{Y})$ & $1.539(\mathrm{Y})$ & $1.458(\mathrm{Y})$ \\
5 & $2.313(\mathrm{Y}+\mathrm{T})$ & $2.105(\mathrm{Y}+\mathrm{T})$ & $1.834(\mathrm{Y})$ & $1.746(\mathrm{X})$ & $1.539(\mathrm{X})$ & $1.458(\mathrm{X})$ \\
6 & $4.115(\mathrm{X})$ & $4.777(\mathrm{Y})$ & $4.432(\mathrm{Y}+\mathrm{T})$ & $4.200(\mathrm{Y}+\mathrm{T})$ & $3.688(\mathrm{Y}+\mathrm{T})$ & $3.520(\mathrm{Y}+\mathrm{T})$ \\
7 & $4.115(\mathrm{Y})$ & $4.777(\mathrm{X})$ & $4.432(\mathrm{X})$ & $4.200(\mathrm{Y}+\mathrm{T})$ & $3.769(\mathrm{Y})$ & $3.607(\mathrm{Y})$ \\
8 & $6.935(\mathrm{Y}+\mathrm{T})$ & $6.281(\mathrm{Y}+\mathrm{T})$ & $4.701(\mathrm{Y}+\mathrm{T})$ & $4.284(\mathrm{X})$ & $3.769(\mathrm{X})$ & $3.607(\mathrm{X})$ \\
9 & $7.879(\mathrm{Y})$ & $8.641(\mathrm{Y})$ & $7.698(\mathrm{Y}+\mathrm{T})$ & $7.005(\mathrm{Y}+\mathrm{T})$ & $6.027(\mathrm{Y}+\mathrm{T})$ & $5.757(\mathrm{Y}+\mathrm{T})$ \\
10 & $7.879(\mathrm{X})$ & $8.641(\mathrm{X})$ & $7.834(\mathrm{Y}+\mathrm{T})$ & $7.508(\mathrm{Y}+\mathrm{T})$ & $6.961(\mathrm{Y}+\mathrm{T})$ & $6.820(\mathrm{Y}+\mathrm{T})$ \\
\hline
\end{tabular}

Table 1: Natural frequencies (in $\mathrm{Hz}$ ) of the diagrid-core system depending on the diagrid diagonal inclination.

After evaluating the natural dynamic response of the diagrid-core system, various harmonic oscillations at different frequencies are applied to the base of the building and the dynamic structural response examined as obtained from equations (2-7). The time-dependent acceleration applied at the base of the building is of the form $u_{\mathrm{g}}{ }^{\prime \prime} \sin \left(\omega_{\mathrm{g}} \mathrm{t}\right)$, being $\mathrm{ug}_{\mathrm{g}}{ }^{\prime \prime}=0.03 \mathrm{~m} / \mathrm{s}^{2}$ the maximum ground acceleration in the $X$ direction and $\omega_{g}=2 \pi / T_{g}$ the ground angular frequency, with $T_{g}$ varying in the range $0.01 \mathrm{~s}-8 \mathrm{~s}$. Figures $2-6$ show the results in terms of maximum story displacement, maximum story velocity, maximum story acceleration, maximum interstory drift and maximum base shear, respectively, as a function of the applied ground oscillation period $\mathrm{T}_{\mathrm{g}}$ and diagrid model.

Figure 2 shows that each diagrid-core system exhibits the largest value of the lateral displacement when the period of the ground oscillation matches the fundamental period of vibration of the building. With reference to the frequency values reported in table 1 , this is found to occur when $\mathrm{T}_{\mathrm{g}}$ is approximately equal to $4.1 \mathrm{~s}, 3.1 \mathrm{~s}, 2.8 \mathrm{~s}, 2.8 \mathrm{~s}, 2.9 \mathrm{~s}$, and $3.1 \mathrm{~s}$ for models 1 , $2,3,4,5$ and 6 , respectively. Therefore the high peaks in the graphs of figure 1 refer to resonance phenomena related to the first modes of vibrations of the diagrid-core system. Clearly, adopting different diagonal inclinations makes the position of this resonance peak to translate horizontally with respect to the variable $T_{g}$, as the fundamental frequency of vibration of the system changes (table 1). It is also interesting to notice that the amplitude of this resonance peak is maximum for model 1 (blue curve), while it reaches a minimum value for models 3 (orange curve) and 4 (purple curve). This result is again in agreement with the abovementioned fact that models 3 and 4 are indeed the most rigid ones against lateral forces, whereas model 1 is the most flexible. Therefore, the maximum dynamic displacements of the building floors are the highest for model 1, lowest for models 3 and 4, and exhibit intermediate values for models 2 (red curve), 5 (green curve) and 6 (light blue curve).

Figures 3 and 4 report the corresponding spectra of maximum story velocities and accelerations, respectively. Again, we find the largest resonance peaks in correspondence of the fundamental periods of the structure. However, in these cases we also start to see additional resonance peaks at lower periods (higher frequencies). The velocity response spectrum shows secondary resonance peaks around $0.5-0.7 \mathrm{~s}$ (figure 3 ), which correspond to the activation of the second lateral vibrational modes (table 1). These secondary peaks are also found in the acceleration response spectrum (figure 4).

This spectrum is also found to exhibit tertiary resonance peaks around $0.2-0.3 \mathrm{~s}$, which correspond to the resonance conditions along the third modes of vibration along the $\mathrm{X}$ direction (table 1). The amplitudes of the resonance peaks in the velocity spectrum (figure 3 ) exhibit similar trends of those reported in the displacement spectrum (figure 2), but here the 
difference between the peaks corresponding to the different diagrid models is reduced. In the acceleration spectrum (figure 4), the amplitudes of the fundamental resonance peaks get somewhat inverted, since model 1 is the one experiencing the lowest acceleration, whereas models 3 and 4 are the ones undergoing the highest value. This is mainly due to the fact that, being models 3 and 4 the stiffest ones, they are not highly deformable but can undergo high accelerations due to their stiffer response.

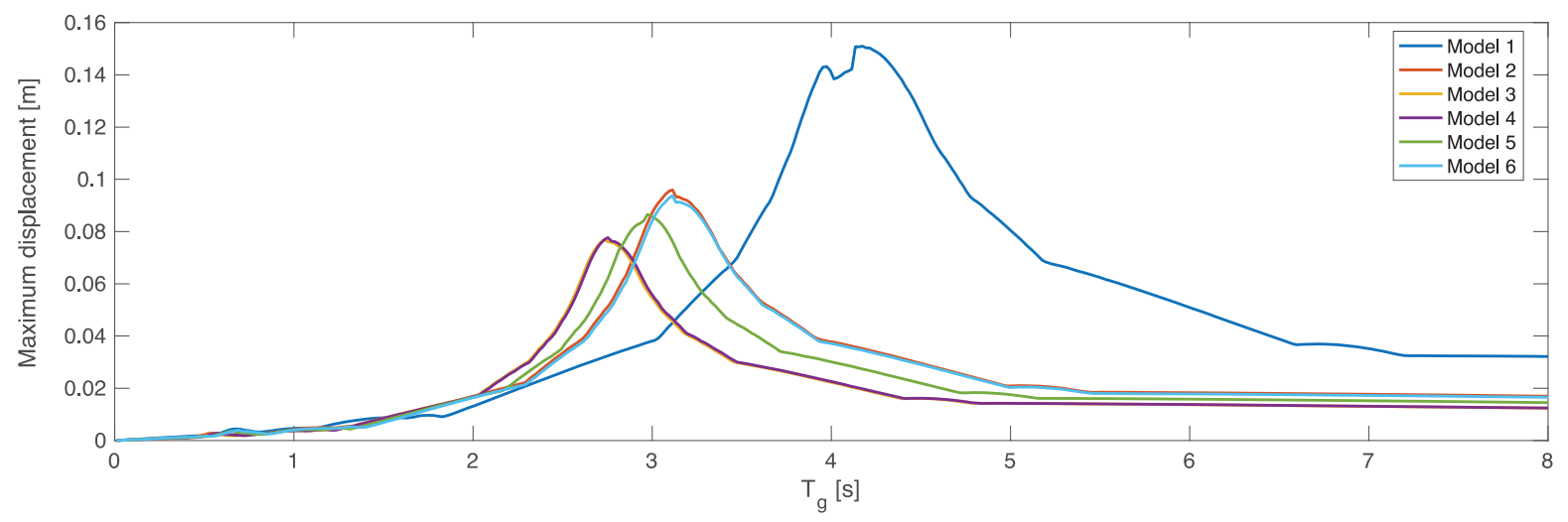

Figure 2: Maximum story displacement of the diagrid-core system.

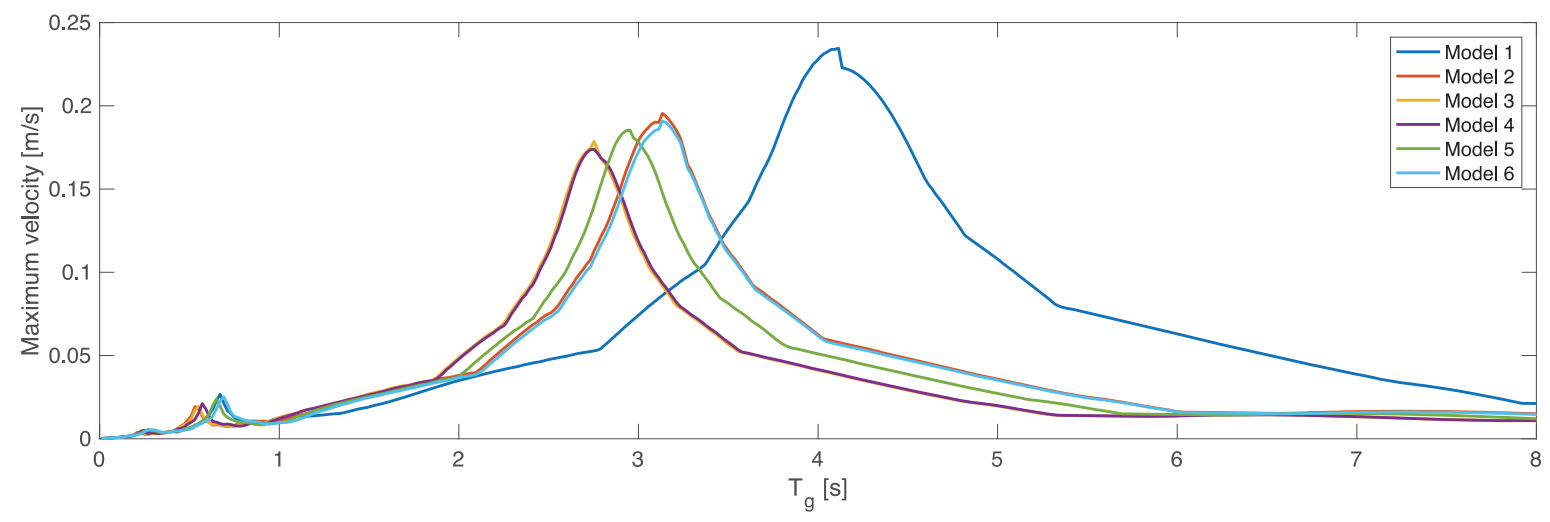

Figure 3: Maximum story velocity of the diagrid-core system.

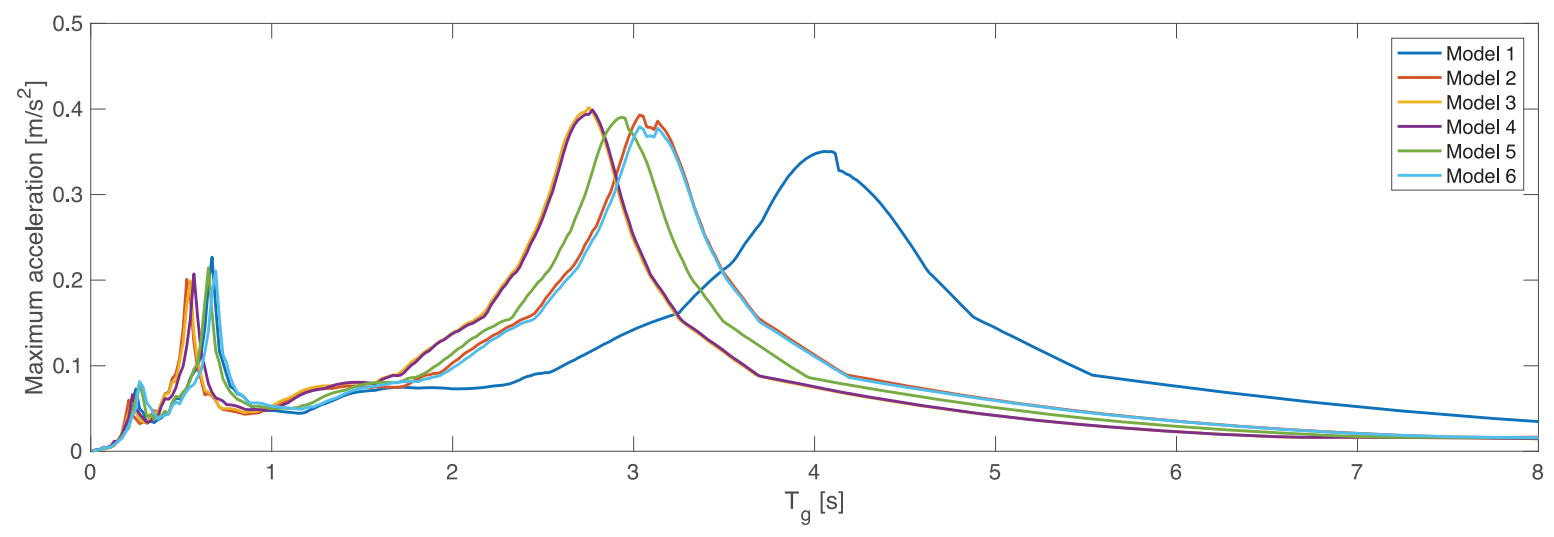

Figure 4: Maximum story acceleration of the diagrid-core system. 
Figure 5 shows the maximum inter-story drifts depending on the diagrid-core model and the value of the ground oscillation period $\mathrm{T}_{\mathrm{g}}$. As can be seen, this spectrum strictly resembles the one of absolute displacements. However, in this case a somewhat clearer occurrence of the second resonance peaks at $0.5-0.7 \mathrm{~s}$ can be observed.

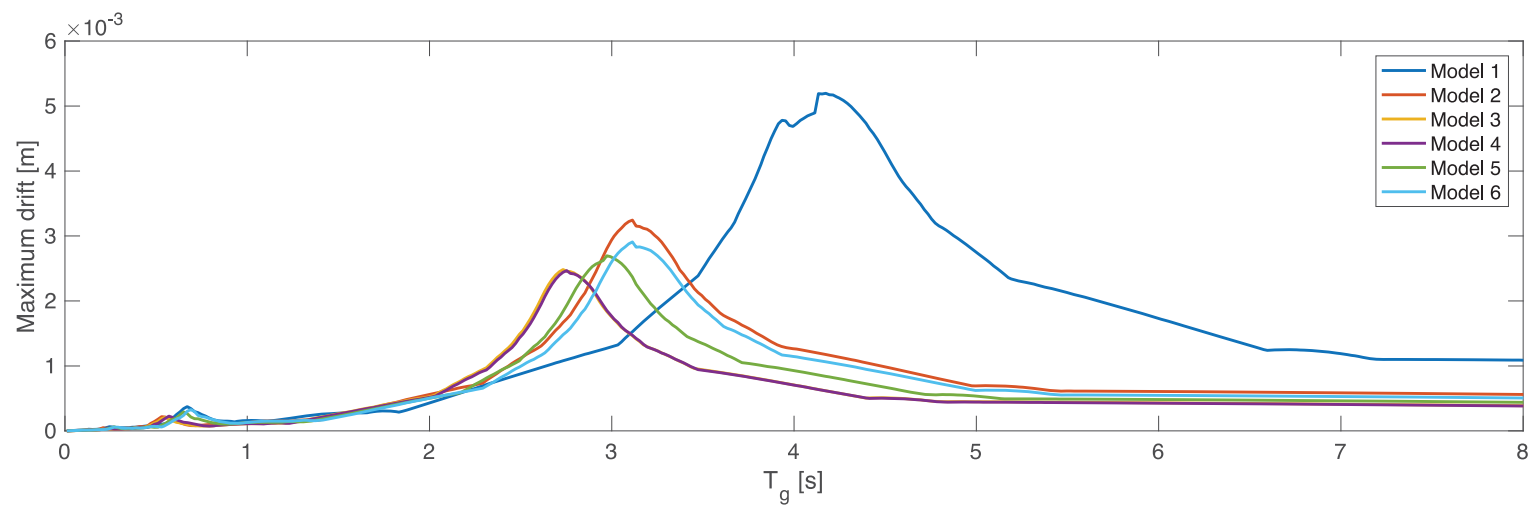

Figure 5: Maximum inter-story drift of the diagrid-core system.

Finally, figure 6 reports the maximum value of the total shear at the base of the building obtained for each oscillation period $\mathrm{T}_{\mathrm{g}}$ and each diagrid-core model. In this case, three clear resonance peaks can again be observed, in analogy with the acceleration spectrum reported in figure 3. From the graph of the base shears, it can be seen that model 1 is the one absorbing the least amount of elastic force, as it is already known to the most flexible under lateral loads. On the other hand, since the other models are stiffer, they exhibit a higher value of the base shear. These results reflect the fact that, under dynamic excitations, there is a general trade-off between the displacement-based and force-based demand. Stiffer structures obviously lead to lower displacements but they usually are required to withstand higher amounts of force. On the other hand, more flexible solutions undergo larger displacements but they are less loaded. However, this is also strongly affected by the frequency of the external excitation. Large dynamic responses, with high dynamic amplifications, are obviously generated if the excitation frequency is close to the fundamental ones of the structure. Conversely, if we are far from the resonance condition, the dynamic response of the building might lead to low values of the displacements as well as elastic forces.

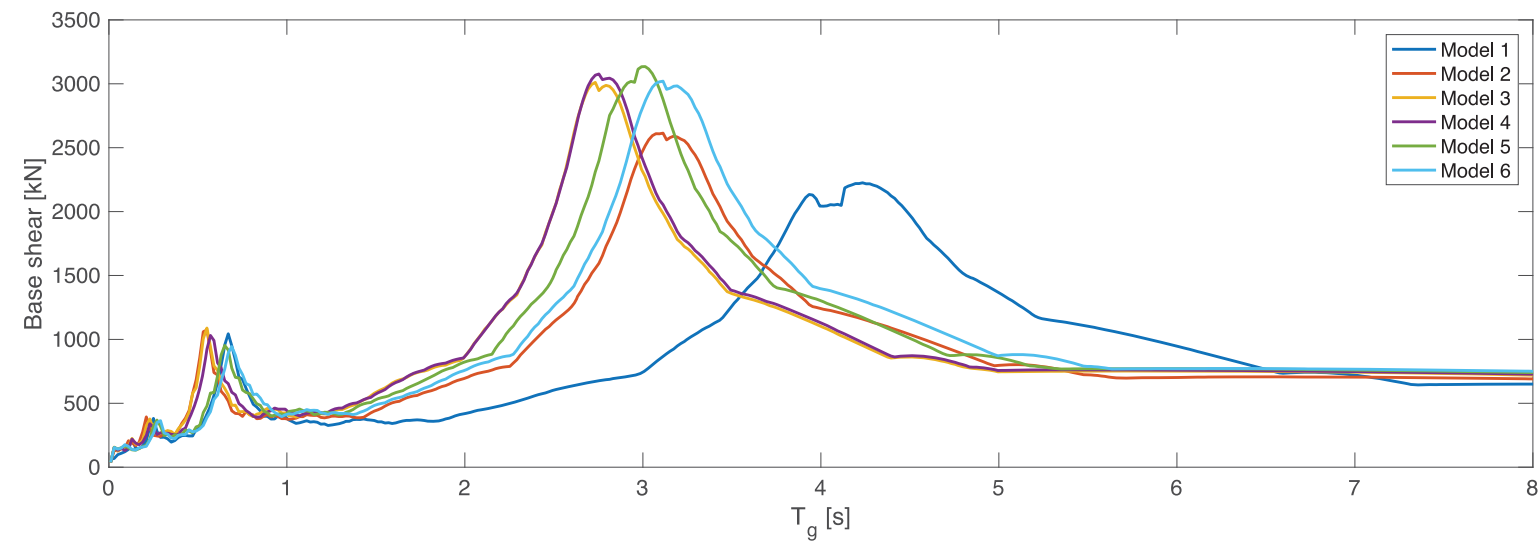

Figure 6: Maximum base shear of the diagrid-core system. 


\section{CONCLUSIONS}

In this contribution we presented the analytical investigation of the dynamic response of a diagrid-core system for tall buildings. For this purpose, a matrix-based method (MBM) was employed within the framework of a General Algorithm (GA). For a set of diagrid-core systems with different inclinations of the external diagonals, the analytical formulation allowed to quickly evaluate the natural frequencies and mode shapes of the structure, as well as the damped response under harmonic oscillations at different frequencies. The semi-analytical formulation led to the investigation of the maximum values of the story displacements, velocities and accelerations under the external excitations, as well as the inter-story drifts and generated base shears.

It was found that diagrid solutions with very shallow diagonals lead to the highest lateral flexibility and lowest torsional deformability. Conversely, the lowest lateral flexibility was found for diagonal inclinations close to $70^{\circ}$. These diagrid solutions led to the lowest values of lateral displacements, but they were the ones experiencing the highest amount of elastic shear force in resonance conditions. Conversely, the most flexible solutions (with very shallow diagonals) were found to undergo the highest dynamic displacements, but experienced the least amount of elastic force. From these considerations, it follows that the dynamic response of a diagrid-core tall building strongly depends on the geometry of the external diagrid tube. However, great attention must also be paid to the fundamental frequency of the external excitation. When far from resonance conditions, each structural solution might have its advantages in terms of minimizing the lateral displacements and generated elastic forces.

\section{REFERENCES}

[1] M.M. Ali, K.S. Moon, Advances in structural systems for tall buildings: Emerging developments for contemporary urban giants. Buildings, 8, 104, 2018.

[2] E. Asadi, H. Adeli, Diagrid: An innovative, sustainable, and efficient structural system. Structural Design of Tall and Special Buildings, 26, e1358, 2017.

[3] D. Scaramozzino, G. Lacidogna, A. Carpinteri, New trends towards enhanced structural efficiency and aesthetic potential in tall buildings: The case of diagrids. Applied Sciences, 10, 3917, 2020.

[4] G. Angelucci, F. Mollaioli, R. Tardocchi, A new modular structural system for tall buildings based on tetrahedral configuration. Buildings, 10, 240, 2020.

[5] K.S. Moon, J.J. Connor, J.E. Fernandez, Diagrid structural systems for tall buildings: Characteristics and methodology for preliminary design. Structural Design of Tall and Special Buildings, 16, 205-230, 2007.

[6] E. Mele, M. Toreno, G. Brandonisio, A. De Luca, Diagrid structures for tall buildings: Case studies and design considerations. Structural Design of Tall and Special Buildings, 23, 124-145, 2014.

[7] C. Liu, K. Ma, Calculation model of the lateral stiffness of high-rise diagrid tube structures based on the modular method. Structural Design of Tall and Special Buildings, 26, e1333, 2017.

[8] G. Lacidogna, D. Scaramozzino, A. Carpinteri, A matrix-based method for the structural analysis of diagrid systems. Engineering Structures, 193, 340-352, 2019. 
[9] A. Carpinteri, G. Lacidogna, S. Puzzi, A global approach for the three-dimensional analysis of tall buildings. Structural Design of Tall and Special Buildings, 19, 518-536, 2010.

[10] A. Carpinteri, G. Lacidogna, G. Nitti, Open and closed shear-walls in high-rise structural systems: Static and dynamic analysis. Curved and Layered Structures, 3, 154-171, 2016.

[11] G. Nitti, G. Lacidogna, A. Carpinteri, Tall buildings subjected to horizontal loading: Analysis of two case studies by an in-house numerical code. Proceedings of the $23 \mathrm{rd}$ Conference of the Italian Association of Theoretical and Applied Mechanics (AIMETA), Salerno, Italy, September 4-7, 292-300, 2017.

[12] G. Nitti, G. Lacidogna, A. Carpinteri, Structural Analysis of High-rise Buildings under Horizontal Loads: A Study on the Piedmont Region Headquarters Tower in Turin. The Open Construction and Building Technology Journal, 13, 81-96, 2019.

[13] G. Lacidogna, G. Nitti, D. Scaramozzino, A. Carpinteri, Diagrid systems coupled with closed- and open-section shear walls: Optimization of geometrical characteristics in tall buildings. Procedia Manufacturing, 44, 402-409, 2020.

[14] K. S. Moon, Optimal grid geometry of diagrid structures for tall buildings. Architectural Science Review, 51, 239-251, 2008.

[15] G. Angelucci, F. Mollaioli, Diagrid structural systems for tall buildings: Changing pattern configuration through topological assessments. Structural Design of Tall and Special Buildings, 26, e1396, 2017.

[16] V. Tomei, M. Imbimbo, E. Mele, Optimization of structural patterns for tall buildings: The case of diagrid. Engineering Structures, 171, 280-297, 2018.

[17] G. Angelucci, F. Mollaioli, Voronoi-like grid systems for tall buildings. Frontiers in the Built Environment, 4, 1-20, 2018.

[18] G. Angelucci, F. Mollaioli, O. A. Shawa, Evaluation of optimal lateral resisting systems for tall buildings subject to horizontal loads. Procedia Manufacturing, 44, 457-464, 2020 . 